\title{
UNIFORM BOUNDS ON BIORTHOGONAL FUNCTIONS FOR REAL EXPONENTIALS WITH AN APPLICATION TO THE CONTROL THEORY OF PARABOLIC EQUATIONS*
}

\author{
BY
}

H. O. FATTORINI (University of Buenos Aires and University of California, Los Angeles)

AND

D. L. RUSSELL (University of Wisconsin, Madison)

1. Introduction and statement of principal results. Let $\Lambda=\left\{\lambda_{k}\right\}$ denote a sequence of positive numbers, $0<\lambda_{1}<\lambda_{2}<\cdots$, let $0<T \leq \infty$ and let $E(\Lambda, T)$ be the closed subspace of $L^{2}(0, T)$ spanned by the functions

$$
p_{k}(t)=\exp \left(-\lambda_{k} t\right) \quad(k=1,2, \cdots) .
$$

It is well known (see, e.g., [10]) that if

$$
\sum_{k=1}^{\infty} \frac{1}{\lambda_{k}}<\infty
$$

then $E(\Lambda, T)$ is a proper subspace of $L^{2}(0, T)$ and, moreover, if we designate by $E(n, \Lambda, T)$ the closed subspace of $E(\Lambda, T)$ spanned by the functions $p_{k}, k \neq n$, then

$$
p_{n} \notin E(n, \Lambda, T) .
$$

If $r_{n} \in E(n, \Lambda, T)$ is the unique element such that

then the functions

$$
\left\|p_{n}-r_{n}\right\|_{L^{2}(0, T)}=\min _{r \in E(n, \Lambda, T)}\left\|p_{n}-r\right\|_{L^{2}(0, T)}
$$

$$
q_{n}=\frac{p_{n}-r_{n}}{\left\|p_{n}-r_{n}\right\|_{L^{2}(0, T)}^{2}}
$$

all lie in $E(\Lambda, T)$ and the sequence $\left\{q_{n}\right\}$ is a biorthogonal sequence for $\left\{p_{n}\right\}$, that is

$$
\begin{aligned}
\left(q_{n}, p_{k}\right)_{L^{2}(0, T)}=\delta_{n k} & =1, & & n=k, \\
& =0, & & n \neq k .
\end{aligned}
$$

This is clear from (1.3), which implies that $\left(q_{n}, r\right)_{L^{2}(0, T)}=0$ for all $r \in E(n, \Lambda, T)$ and from the fact that

$$
\begin{aligned}
\left(q_{n}, p_{n}\right)_{L^{2}(0, T)} & =\left(q_{n}, p_{n}\right)_{L^{2}(0, T)}-\left(q_{n}, r_{n}\right)_{L^{2}(0, T)} \\
& =\left(\frac{p_{n}-r_{n}}{\left\|p_{n}-r_{n}\right\|_{L^{2}(0, T)}^{2}}, p_{n}-r_{n}\right)_{L^{2}(0, T)}=1 .
\end{aligned}
$$

* Received November 21, 1972. The research reported in this paper was supported by the National Science Foundation (grant GP-9658), the Office of Naval Research (contract NR 041-404) and the Department of the Army (contract DA-31-124-ARO-D-462). 
It is of considerable interest for various applications (one of which is described in the final section of this paper) to be able to estimate the norm of $q_{n}$ :

$$
\left\|q_{n}\right\|_{L^{2}(0, T)}=\frac{1}{\left\|p_{n}-r_{n}\right\|_{L^{2}(0, T)}} .
$$

A number of results of this type are already available for individual sequences $\Lambda$ satisfying certain additional restrictions. Luxemburg and Korevaar [8] have shown that for complex $\left\{\lambda_{k}\right\}$ satisfying

$$
\operatorname{Re} \lambda_{n} \geq \delta\left|\lambda_{n}\right|, \quad\left|\lambda_{m}-\lambda_{n}\right| \geq|m-n| \rho
$$

( $\delta$ and $\rho$ positive constants) and $\sum_{k=1}^{\infty}\left(1 /\left|\lambda_{n}\right|\right)<\infty$ one has

$$
\left\|q_{n}\right\|_{L^{2}(0, T)} \leq \exp \left(\epsilon \operatorname{Re} \lambda_{n}\right) \quad(n=1,2, \cdots)
$$

for any $\epsilon>0$ provided that $n \geq N, N$ sufficiently large. Also, the present authors have shown in an earlier paper [6] that if the $\lambda_{n}$ are real and satisfy the asymptotic relationship

$$
\lambda_{n}=K(n+\alpha)^{\beta}+o\left(n^{\beta-1}\right) \quad(n \rightarrow \infty)
$$

where $K>0, \beta>1$ and $\alpha$ is real, then there exist constants $\hat{K}>0, K_{\beta}>0$ such that

$$
\left\|q_{n}\right\|_{L^{2}(0, \infty)} \leq \hat{K} \exp \left[\left(K_{\beta}+o(1)\right) \lambda_{n}{ }^{1 / \beta}\right] \quad(n \geq 1)
$$

where $o(1)$ indicates a term tending to 0 as $n$ tends to infinity. The constant $K_{\beta}$ is explicitly computed in [6].

The proofs of the above results do not yield much, if any, information on the way in which the constants $\hat{K}, K_{\beta}$, depend on the particular sequence $\Lambda$ in question. In this paper we present results which are "uniform" in that the constants which appear in the formulae for estimation of $\left\|q_{n}\right\|_{L^{2}(0, T)}$ are independent of the particular sequence $\Lambda$, provided that $\Lambda$ is taken to lie in a class of sequences satisfying certain properties. Thus these constants depend only upon the conditions imposed on $\Lambda$ rather than upon the particular sequence $\Lambda$.

As in [6], we begin by studying the problem formulated above in the special case where $T=+\infty$. The first result, proved in Sec. 2, is

Theorem 1.1. Let $\rho$ be a positive number and let $\mathfrak{N}(\epsilon)$ be a positive integer-valued function defined for $\epsilon>0$. Denote by $\&(\rho, \mathfrak{T})$ the class of all sequences of positive numbers $\Lambda=\left\{\lambda_{n}\right\}$ that satisfy the conditions

$$
\begin{array}{cl}
\lambda_{1} \geq \rho, & \lambda_{n+1}-\lambda_{n} \geq \rho \quad(n=1,2, \cdots), \\
& \sum_{n=\mathfrak{N}(\epsilon)}^{\infty} \frac{1}{\lambda_{n}} \leq \epsilon, \quad \epsilon>0 .
\end{array}
$$

Then there is a positive function $K(\epsilon)$, defined for $\epsilon>0$ and determined solely by $\rho$ and $\Re$, such that

$$
\left\|q_{n}\right\|_{L^{2}(0, \infty)} \leq K(\epsilon) \exp \left(\epsilon \lambda_{n}\right), \quad(n=1,2, \cdots)
$$

where $\left\{q_{n}\right\}$ is the biorthogonal sequence for $\left\{p_{k}\right\}$ ( $p_{k}$ given by (1.1)) described above and $\Lambda$ is any sequence in $\mathscr{L}(\rho, \mathfrak{N})$.

We remark that $\Re$ would ordinarily be taken to be a decreasing function of $\epsilon$ satisfying $\lim _{\epsilon \downarrow 0} \Re(\epsilon)=\infty$. 
A result more precise than the foregoing can be obtained if we impose more stringent requirements on the sequence $\Lambda$. This is done in Secs. 3 and 4 , where we prove

Theorem 1.2. Let the function $F$ be convex and strictly increasing on $[0, \infty)$, with

$$
F(0)=0, \quad \int_{1}^{\infty} \frac{d v}{F(v)}<\infty
$$

and let $G$ be the inverse of $F$, i.e. $G(F(v)) \equiv v, v \in[0, \infty)$. Assume that for some constant $C>0$

$$
G(u v) \leq C G(u) G(v) \quad(0 \leq u, v<\infty) .
$$

Finally, let $l$ be a positive constant. We denote by $\mathfrak{N}(F, l)$ the class of all sequences of positive numbers $\Lambda=\left\{\lambda_{k}\right\}$ which satisfy

$$
\begin{gathered}
w_{1}=G\left(\lambda_{1}\right) \geq l, \\
w_{k+1}-w_{k}=G\left(\lambda_{k+1}\right)-G\left(\lambda_{k}\right) \geq l \quad(k=1,2, \cdots) .
\end{gathered}
$$

Then there is a constant $K$, determined solely by $l$ and $F$ such that

$$
\left\|q_{n}\right\|_{L^{2}(0, \infty)} \leq \exp \left[K G\left(\lambda_{n}\right)\right] \quad(n=1,2, \cdots)
$$

where $\left\{q_{n}\right\}$ is the biorthogonal sequence for $\left\{p_{k}\right\}$ described at the beginning of this section and $\Lambda$ is any sequence in $\pi(F, l)$.

Once these uniform results have been obtained in the case $T=\infty$ one can obtain corresponding results for $T<\infty$ rather simply by means of a "uniform" version of a theorem of Schwartz [10] on "comparison of norms". We describe briefly how this is done. Let $p_{k}$, as in (1.1), be thought of as an element of $L^{2}(0, \infty)$, and let $\hat{p}_{k}$ denote the restriction of $p_{k}$ to the interval $[0, T]$, where $T$ is a fixed positive number, $0<T<\infty$. Clearly, $\hat{p}_{k} \in L^{2}[0, T]$. The restriction operator $R: L^{2}(0, \infty) \rightarrow L^{2}(0, T)$ is bounded (by 1) and maps linear combinations of the $\left\{p_{k}\right\}$ into linear combinations of the $\left\{\hat{p}_{k}\right\}$. Then it is clear that its restriction $S$ to $E(\Lambda, \infty)$ maps this space into $E(\Lambda, T)$ (and, as it is easy to see, with norm 1 ). The above mentioned result of Schwartz enables us to conclude that $S$ has a bounded inverse whenever (1.2) holds. Our "uniformization" of this result is

Theorem 1.3. Let $T(0<T<\infty)$ be fixed and let $\Lambda$ be a sequence in a class $\mathscr{L}(\rho, \mathfrak{T})$ as described in Theorem 1 . Then the operator $S$ maps $E(\Lambda, \infty)$ onto $E(\Lambda, T)$ in a one-to-one fashion and thus has an inverse $S^{-1}: E(\Lambda, T) \rightarrow E(\Lambda, \infty)$. Moreover, there is a positive constant $B$ determined solely by $\rho, \mathfrak{N}$ and $T$ such that

$$
\left\|S^{-1}\right\| \leq B
$$

for any sequence $\Lambda \in \mathfrak{L}(\rho, \mathfrak{X})$.

The proof will be given in Sec. 5. Note that Theorem 1.3 does not contain Schwartz' result as the "separation condition" (1.8) is not used by Schwartz.

We note next that if $\Re(F, l)$ is a class of sequences as described in Theorem 1.2, then $\mathfrak{T}(F, l)$ is included in one of the classes $\mathcal{L}(\rho, \mathfrak{N})$ of Theorem 1 . In fact, we may take $\rho=F(l)$. For, since $F$ is increasing, $\lambda_{1}=F\left(w_{1}\right) \geq F(l)=\rho$. On the other hand, let $f$ be the function whose graph is a straight line through $\left(w_{k}, F\left(w_{k}\right)\right)$ and $\left(w_{k+1}\right.$, $\left.F\left(w_{k+1}\right)\right)(k \geq 1)$, i.e. 


$$
f(v)=F\left(w_{k}\right)+\left(\frac{F\left(w_{k+1}\right)-F\left(w_{k}\right)}{w_{k+1}-w_{k}}\right)\left(v-w_{k}\right)
$$

and let

$$
\tilde{f}(v)=\frac{F(l)}{l} v
$$

which passes through $(0,0)=(0, F(0))$ and $(l, F(l))$. Since $F$ is convex and $0<l<w_{k}$,

$$
f(0) \leq F(0)=\tilde{f}(0), \quad \tilde{f}\left(w_{k}\right) \leq F\left(w_{k}\right)=f\left(w_{k}\right) .
$$

Since $f$ and $\tilde{f}$ are both affine functions, the above inequalities imply that

$$
f^{\prime}(v) \equiv \frac{F\left(w_{k+1}\right)-F\left(w_{k}\right)}{w_{k+1}-w_{k}} \geq \tilde{f}^{\prime}(v) \equiv \frac{F(l)}{l}=\frac{\rho}{l} .
$$

Then the inequality $w_{k+1}-w_{k} \geq l$ implies $F\left(w_{k+1}\right)-F\left(w_{k}\right) \geq \rho$, i.e. $\lambda_{k+1}-\lambda_{k} \geq \rho$ $(k=1,2, \cdots)$. To find $\mathfrak{N}(\epsilon)$, we note that, for $n \geq 2$,

$$
\sum_{k=n}^{\infty} \frac{1}{\lambda_{k}}=\sum_{k=n}^{\infty} \frac{1}{F\left(w_{k}\right)} \leq \sum_{k=n}^{\infty} \frac{1}{w_{k}-w_{k-1}} \int_{w_{k-1}}^{w_{k}} \frac{d v}{F(v)} \leq \frac{1}{l} \int_{w_{n-1}}^{\infty} \frac{d v}{F(v)} .
$$

From (1.18) we see that we may take $\Re(\epsilon)$ to be the least integer $n$ for which $n \geq 2$ and

$$
\frac{1}{l} \int_{w_{n-1}}^{\infty} \frac{d v}{F(v)} \leq \epsilon
$$

Accordingly, we have

Corollary 1.4. The result of Theorem 3 is valid for sequences $\Lambda$ in a class $\operatorname{TN}(F, l)$ as described in Theorem 2; the constant $B$ in (1.17) is determined solely by $F, l$ and $T$.

We proceed now to the construction of biorthogonal sequences in $L^{2}(0, T)$. Since both $E(\Lambda, \infty)$ and $E(\Lambda, T)$ are Hilbert spaces, the adjoint $S^{*}: E(\Lambda, T) \rightarrow E(\Lambda, \infty)$ is well defined; moreover,

$$
\left\|\left(S^{-1}\right)^{*}\right\|=\left\|S^{-1}\right\| \leq B(=B(\rho, \mathfrak{T}))
$$

if $\Lambda$ lies in a class $\mathscr{L}(\rho, \mathfrak{T})$. Since

$$
\begin{aligned}
\left(\left(S^{-1}\right)^{*} q_{n}, \hat{p}_{k}\right)_{L^{2}(0, T)} & =\left(q_{n}, S^{-1} \hat{p}_{k}\right)_{L^{2}(0, \infty)} \\
& \left.=\left(q_{n}, p_{k}\right)_{L^{2}(0, \infty)}\right)=\delta_{n k},
\end{aligned}
$$

we conclude that the sequence $\left\{\hat{p}_{k}\right\}$ has a biorthogonal sequence $\left\{\hat{q}_{n}\right\}$ in $E(\Lambda, T)$ given by $\hat{q}_{n}=\left(S^{-1}\right)^{*} q_{n}(n=1,2, \cdots)$. Inequality (1.19) implies

$$
\left\|\hat{q}_{n}\right\|_{L^{2}(0, T)} \leq B\left\|q_{n}\right\|_{L^{2}(0, \infty)} .
$$

Thus we have proved

Theorem 1.5. The results of Theorems 1.1 and 1.2 remain valid with $L^{2}(0, \infty)$ replaced by $L^{2}(0, T)(0<T<\infty)$ provided that

a) In Theorem 1.1, inequality (1.10) becomes

$$
\left\|\hat{q}_{n}\right\|_{L^{2}(0, T)} \leq B K(\epsilon) \exp \left(\epsilon \lambda_{n}\right) \quad(n=1,2, \cdots) ;
$$


b) In Theorem 1.2, inequality (1.16) is replaced by

$$
\left\|\hat{q}_{n}\right\|_{L^{2}(0, T)} \leq B \exp \left(K G\left(\lambda_{n}\right)\right) \quad(n=1,2, \cdots) .
$$

It should be noted that the biorthogonal elements $q_{n}$ of Theorems 1 and 2 lie in $E(\Lambda, \infty)$; consequently, the biorthogonal elements $\hat{q}_{n}$ in Theorem 4 lie in $E(\Lambda, T)$. This implies that $q_{n}$ and $\hat{q}_{n}$ have minimal norms in $L^{2}(0, \infty)$ and $L^{2}(0, T)$, respectively, as compared with other possible biorthogonal elements for the sequences $\left\{p_{k}\right\}$ and $\left\{\hat{p}_{k}\right\}$ respectively. Thus, while improvements over the bounds obtained in Theorems 1,2 and 4 are not ruled out, such improvements cannot arise out of a different specification of the biorthogonal elements. (Since $E(\Lambda, \infty)^{\perp}$ and $E(\Lambda, T)^{\perp}$ are both known to be infinitedimensional in $L^{2}(0, \infty)$ and $L^{2}(0, T)$, respectively, there are infinitely many possible biorthogonal systems for $\left\{p_{k}\right\}$ and $\left\{\hat{p}_{k}\right\}$, respectively.)

We note also that when working in $L^{2}(0, \infty)$ we need $\lambda_{k}>0$ to ensure that $p_{k}$ lies in $L^{2}(0, \infty)$. This is no longer necessary in $L^{2}(0, T)$; on the other hand-as will be seen in Sec. 6-the condition $\lambda_{k}>0$ is an inconvenient restriction in some applications. Let us consider, then, a sequence $\tilde{\Lambda}=\left\{\tilde{\lambda}_{k}\right\}$ with the property that $\tilde{\Lambda}+l_{0}=\left\{\tilde{\lambda}_{k}+l_{0}\right\}$ satisfies (1.8) and (1.9) or (1.14) and (1.15), that is, lies in a class $\&(\rho, \Re)$ or a class $\Re(F, l)$. We define $E(\tilde{\Lambda}, T)$ as we $\operatorname{did} E(\Lambda, T)$ above. Let $\lambda_{k} \equiv \tilde{\lambda}_{k}+l_{0}$ and

$$
\hat{p}_{k}(t)=\exp \left(-\left(\tilde{\lambda}_{k}+l_{0}\right) t\right)=\exp \left(-\lambda_{k} t\right) \quad(0 \leq t \leq T, k \geq 1) .
$$

Theorem 4 yields bounds on the norms of biorthogonal elements $\hat{q}_{n}$ for this sequence in $L^{2}(0, T)$. It is very simple to verify that the functions $\tilde{q}_{n}(t)=\hat{q}_{n}(t) \exp \left(-l_{0} t\right)(0 \leq t \leq$ $T, n \geq 1)$ are biorthogonal to the functions $\tilde{p}_{k}(t)=\exp \left(-\tilde{\lambda}_{k} t\right)(k \geq 1)$ in $L^{2}(0, T)$. Since $\left|\exp \left(-l_{0} t\right)\right| \leq 1$ for $t \geq 0$, we obtain immediately the following corollary to Theorem 1.4.

Corollary 1.6. Let $\tilde{\mathscr{L}}(\rho, \mathfrak{N})$ and $\tilde{\mathfrak{M}}(F, l)$ denote classes of sequences $\tilde{\Lambda}$ such that $\tilde{\Lambda}+l_{0}$ lies in $\mathfrak{L}(\rho, \mathfrak{N})$ or $\mathfrak{T}(F, l)$. respectively. The real number $l_{0}$ is assumed non-negative but may be different for different sequences $\tilde{\Lambda}$. Then, for $n=1,2, \cdots$ the biorthogonal elements $\tilde{q}_{n}$ satisfy

$$
\begin{aligned}
& \left\|\hat{q}_{n}\right\|_{L^{2}(0, T)} \leq B K(\epsilon) \exp \left[\epsilon\left(\tilde{\lambda}_{n}+l_{0}\right)\right], \quad \tilde{\Lambda} \in \mathcal{L}(\rho, \mathfrak{T}) . \\
& \left\|\tilde{q}_{n}\right\|_{L^{2}(0, T)} \leq B \exp \left[K G\left(\tilde{\lambda}_{n}+l_{0}\right)\right], \quad \tilde{\Lambda} \in \mathfrak{L}(F, l) .
\end{aligned}
$$

where $K(\epsilon), K$ and $B$ are as described in Theorems 1, 2 and 3 .

There is no reason to suppose that Corollary 6 is the best possible in any sense.

We shall apply in Sec. 6 the results herein described - specifically, inequality (1.23)to solve a boundary controllability problem for the heat equation in a $n$-dimenslonal sphere. It turns out that we will need to deduce an estimate of the type of (1.23), but for the $L^{\infty}(0, T)$-norm of $\tilde{q}_{n}$ instead of the $L^{2}(0, T)$-norm. This can be obtained by an almost direct application of Corollary 1.6. We refer the reader to Sec. 6 for statements and proofs of these estimates as well as for a precise description of the control problem. We note that the results contained in Sec. 2 are not used for the control problem.

In addition to the other results mentioned earlier, we also refer the reader to the paper of Mizel and Seidman [9], who have obtained the uniform result expressed in our Theorem 1.1 using essentially the hypotheses of our Theorem 1.2 with $P(x)=x^{\beta}, \beta>1$. Moreover, our proof of Theorem 1.3 utilizes the same method which they employed in their proof of the analogous assertion in [9]. They also give application of this result to 
parabolic partial differential equations. Indded, they study an observability problem which is dual, in a certain sense, to the control problem we treat in Sec. 6 .

2. Proof of Theorem 1.1. We make use of (1.5) combined with the explicit calculation of $\left\|p_{n}-r_{n}\right\|$ carried out by Kaczmarz and Steinhaus [7]. According to them,

$$
\left\|q_{n}\right\|_{L^{2}(0, \infty)}=\left(\frac{\lambda_{n}}{2}\right)^{1 / 2} \frac{\prod_{i=1}^{\infty}\left[1+\left(\lambda_{n} / \lambda_{i}\right)\right]}{\prod_{i=1}^{\infty}\left|1-\left(\lambda_{n} / \lambda_{i}\right)\right|}
$$

where $\Pi^{\prime}$ indicates that $j \neq n$ in the infinite product.

The estimation of the infinite product in the numerator of (2.1) is trivial. Given $\hat{\epsilon}>0$,

$$
\prod_{i=1}^{\infty}\left(1+\frac{\lambda_{n}}{\lambda_{j}}\right)=\prod_{i=1}^{\Re(\hat{\hat{e}})-1}\left(1+\frac{\lambda_{n}}{\lambda_{j}}\right) \prod_{i=\Re(\hat{\epsilon})}^{\infty}\left(1+\frac{\lambda_{n}}{\lambda_{j}}\right) .
$$

Since $\lambda_{i} \geq \rho(j \geq 1)$,

$$
\prod_{i=1}^{\Re(\hat{\hat{e}})-1}\left(1+\frac{\lambda_{n}}{\lambda_{i}}\right) \leq\left(1+\frac{\lambda_{n}}{\rho}\right)^{\Re(\hat{\hat{e}})-1}
$$

For the remaining factors we use the inequality $1+x \leq e^{x}$ valid for $x \geq 0$.

$$
\prod_{i=\Re(\hat{\epsilon})}^{\infty}\left(1+\frac{\lambda_{n}}{\lambda_{i}}\right) \leq \prod_{i=\Re(\hat{\epsilon})}^{\infty} \exp \left(\frac{\lambda_{n}}{\lambda_{j}}\right)=\exp \left[\lambda_{n}\left(\sum_{i=\Re(\hat{\epsilon})}^{\infty} \frac{1}{\lambda_{j}}\right)\right] \leq \exp \left(\hat{\epsilon} \lambda_{n}\right)
$$

the last inequality following from (1.9). Thus,

$$
\prod_{i=1}^{\infty}\left(1+\frac{\lambda_{n}}{\lambda_{i}}\right) \leq\left(1+\frac{\lambda_{n}}{\rho}\right)^{\Re(\hat{\hat{\epsilon}})-1} \exp \left(\hat{\hat{\epsilon}} \lambda_{n}\right) \quad(n \geqq 1) .
$$

The estimation of the infinite product in the denominator of (2.1) requires more care We shall use a method that appears in the paper of Luxemburg and Korevaar [8]. Divide a sequence $\Lambda \in \mathfrak{L}(\rho, \Re)$ in three parts, depending on a given integer $n$ :

$$
\begin{aligned}
& \Lambda_{1}(n)=\left\{\lambda_{i} \in \Lambda ; \lambda_{i} \leq \frac{1}{2} \lambda_{n}\right\}, \\
& \Lambda_{2}(n)=\left\{\lambda_{i} \in \Lambda ; \frac{1}{2} \lambda_{n}<\lambda_{i} \leq 2 \lambda_{n}\right\}, \\
& \Lambda_{3}(n)=\left\{\lambda_{i} \in \Lambda ; \lambda_{i}>2 \lambda_{n}\right\} .
\end{aligned}
$$

Plainly,

$$
\prod_{\lambda_{i} \in \Lambda_{1}(n)}\left|1-\frac{\lambda_{n}}{\lambda_{i}}\right| \geq \prod_{\lambda_{i} \in \Lambda_{1}(n)} 1=1
$$

Just as plainly, if $n \geq \mathscr{N}(\hat{\boldsymbol{\epsilon}})$,

$$
\log \left(\prod_{\lambda_{i} \in \Lambda_{3}(n)}\left|1-\frac{\lambda_{n}}{\lambda_{j}}\right|\right)=\sum_{\lambda_{i} \in \Lambda_{3}(n)} \log \left(1-\frac{\lambda_{n}}{\lambda_{j}}\right) \geq-2 \lambda_{n} \sum_{\lambda_{j} \in \Lambda_{3}(n)} \frac{\lambda}{\lambda_{i}} \geq-2 \hat{\epsilon} \lambda_{n},
$$

where we have used the inequality $\log (1-x) \geq-2 x$ (or, equivalently, $e^{-2 x} \leq 1-x$ ) valid at least for $0 \leq x \leq \frac{1}{2}$. Accordingly, 


$$
\prod_{\lambda_{i} \in \Lambda_{3}(n)}\left|1-\frac{\lambda_{n}}{\lambda_{j}}\right| \geq \exp \left(-2 \hat{\epsilon} \lambda_{n}\right)
$$

On the other hand,

$$
\prod_{\lambda_{i} \in \Lambda_{z}(n)}^{\prime}\left|1-\frac{\lambda_{n}}{\lambda_{j}}\right|=\prod_{\lambda_{j} \in \Lambda_{2}(n)}^{\prime}\left|\frac{\lambda_{j}-\lambda_{n}}{\lambda_{i}}\right| \geq \prod_{\lambda_{i} \in \Lambda_{2}(n)}^{\prime} \frac{|j-n| \rho}{2 \lambda_{n}}=r_{n} ! s_{n} !\left(\frac{\rho}{2 \lambda_{n}}\right)^{r_{n}+s_{n}}
$$

where $r_{n}$ (resp. $s_{n}$ ) is the number of $\lambda_{i}$ 's in $\Lambda_{2}(n)$ with $\lambda_{i}\left\langle\lambda_{n}\right.$ (resp. $\lambda_{i}>\lambda_{n}$ ); note that in the deduction of (2.5) we have used the inequality

$$
\left|\lambda_{i}-\lambda_{n}\right| \geq|j-n| \rho
$$

which is an immediate consequence of (1.8). Now,

$$
\frac{r_{n}+s_{n}}{2 \lambda_{n}}=\sum_{\lambda_{i} \in \Lambda_{2}(n)}^{\prime} \frac{1}{2 \lambda_{n}} \leq \sum_{\lambda_{j} \in \Lambda_{2}(n)}^{\prime} \frac{1}{\lambda_{i}} \leq \sum_{\lambda_{j}>\lambda_{n} / 2}^{\prime} \frac{1}{\lambda_{j}}
$$

We wish to show that we can make this last quantity as small as we wish-uniformly for all $\Lambda \in \mathcal{L}(\rho, \mathfrak{l})$-by choosing $n$ sufficiently large. We have

$$
\sum_{\lambda_{i}>\lambda_{n} / 2}^{\prime} \frac{1}{\lambda_{i}}=\sum_{\lambda_{n}>\lambda_{i}>\lambda_{n} / 2}^{\prime} \frac{1}{\lambda_{i}}+\sum_{i>n} \frac{1}{\lambda_{i}}
$$

Let $\delta>0, n>2 \Re(\delta / 5)+2$. Then

$$
\sum_{i>n} \frac{1}{\lambda_{i}} \leq \sum_{i>\vartheta(\delta / 5)} \frac{1}{\lambda_{i}} \leq \delta / 5
$$

and

$$
\sum_{\lambda_{n}>\lambda_{i}>\lambda_{n} / 2} \frac{1}{\lambda_{i}} \leq n\left(\frac{2}{\lambda_{n}}\right)=4 \frac{n}{2} \cdot \frac{1}{\lambda_{n}} \leq 4 \sum_{i=[n / 2]}^{n-1} \frac{1}{\lambda_{i}}<4 \sum_{j=\eta(\delta / 5)}^{\infty} \frac{1}{\lambda_{i}}=\frac{4}{5} \delta .
$$

Combining (2.7) and (2.8) we obtain

$$
\sum_{\lambda_{i}>\lambda_{n} / 2} \frac{1}{\lambda_{i}}<\delta \quad(n \geq 2 \Re(\delta / 5)+2)
$$

for any sequence $\Lambda \in \mathfrak{L}(\rho, \mathfrak{N})$. Going back to (2.6), this implies that

$$
r_{n}=\eta_{n} \lambda_{n}, \quad s_{n}=\nu_{n} \lambda_{n}
$$

where

$$
\lim _{n \rightarrow \infty} \eta_{n}=\lim _{n \rightarrow \infty} \nu_{n}=0
$$

uniformly for $\Lambda \in \mathscr{L}(\rho, \mathfrak{\Re})$.

We recall now Stirling's formula, according to which we have $\log r_{n} !=r_{n} \log r_{n}-r_{n}+$ $\frac{1}{2} \log 2 \pi r_{n}+\beta\left(r_{n}\right)\left(\beta\left(r_{n}\right) \rightarrow 0\right.$ as $\left.r_{n}=\eta_{n} \lambda_{n} \rightarrow \infty\right)$ and we use it to show that

$$
\begin{aligned}
\log \left[r_{n} !\left(\frac{\rho}{2 \lambda_{n}}\right)^{r_{n}}\right]=\log r_{n} !+r_{n} \log \frac{\rho}{2} & -r_{n} \log \lambda_{n} \\
& =r_{n} \log \left(\frac{r_{n} \rho}{2 e \lambda_{n}}\right)+\frac{1}{2} \log 2 \pi r_{n}+\beta\left(r_{n}\right) .
\end{aligned}
$$


A similar calculation applies with $r_{n}$ replaced by $s_{n}$ :

$$
\log \left[s_{n} !\left(\frac{\rho}{2 \lambda_{n}}\right)^{s_{n}}\right]=s_{n} \log \left(\frac{s_{n} \rho}{2 e \lambda_{n}}\right)+\frac{1}{2} \log 2 \pi s_{n}+\beta\left(s_{n}\right) .
$$

From (2.12) and (2.13) we obtain

$$
r_{n} ! s_{n} !\left(\frac{\rho}{2 \lambda_{n}}\right)^{r_{n}+s_{n}}=\left[\left(\frac{\eta_{n} \rho}{2 e}\right)^{\eta_{n}}\left(\frac{\nu_{n} \rho}{2 e}\right)^{\nu_{n}}\right]^{\lambda_{n}} 2 \pi\left(\eta_{n} \nu_{n}\right)^{1 / 2} \lambda_{n} \exp \left[\beta\left(r_{n}\right)+\beta\left(s_{n}\right)\right] .
$$

Combining this with (2.5) and making use of (2.3) and (2.4) we see that, given $\hat{\epsilon}>0$ we can find a constant $K(\hat{\epsilon})>0$ such that

$$
\prod_{i=1}^{\infty}\left|1-\frac{\lambda_{n}}{\lambda_{i}}\right| \geq M(\hat{\epsilon}) \exp \left(-3 \hat{\epsilon} \lambda_{n}\right) \quad(n \geq 1)
$$

for any $\Lambda \in \mathcal{L}(\rho, \mathfrak{T}), M(\hat{\epsilon})$ independent of $\Lambda$. Going back to (2.1) and making use of (2.2), we see that for any $\hat{\epsilon}>0$ there exists $K(\hat{\epsilon})>0$ such that, for any $\Lambda \in \mathfrak{L}(\rho, \mathfrak{T})$,

$$
\left\|q_{n}\right\|_{L^{2}(0, \infty)} \leq\left(\frac{\lambda_{n}}{2}\right)^{1 / 2} K(\hat{\epsilon}) \exp \left(4 \hat{\epsilon} \lambda_{n}\right),
$$

from which the conclusion of Theorem 1.1 follows immediately.

3. Proof of Theorem 1.2. We begin by establishing some simple results concerning the function $F$ and its inverse.

Lemma 3.1.

a) $\lim _{u \rightarrow \infty}(G(u) / u)=0$

b) $\int_{1}^{\infty} G(u) \frac{d u}{u^{2}}<\infty$.

Proof: Convex functions are absolutely continuous [13, p. 24]. This justifies the following change of variables and integration by parts:

$\int_{1}^{\bar{u}} G(u) \frac{d u}{u^{2}}+\frac{G(\bar{u})}{\bar{u}}=\int_{G(1)}^{G(\bar{u})} \frac{v}{F(v)^{2}} F^{\prime}(v) d v+\frac{G(\bar{u})}{\bar{u}}=G(1)+\int_{G(1)}^{(G \bar{u})} \frac{d v}{F(v)} \quad(\bar{u} \geq 1)$.

This inequality shows, taking (1.12) into account, that $\int_{1}{ }^{\tilde{u}} G(u)\left(d u / u^{2}\right)$ is bounded as $\bar{u} \rightarrow \infty$, which implies (3.2). It also shows that $\lim _{\tilde{u} \rightarrow \infty}(G(\bar{u}) / \bar{u})$ exists; if it were not zero, this would contradict (3.2).

The following result shows that the restrictions on $F$ and $G$ in the statement of Theorem 1.2 are quite severe. We note that only the second inequality in (3.4) and the first inequality in (3.5) will be used in the proof of Theorem 1.2.

Lemma 3.2. There exist positive constants $a_{0}, a_{1}, b_{0}, b_{1}, c_{0}, c_{1}$ such that

$$
\begin{array}{ll}
b_{0} u^{a_{0}} \leq G(u) \leq b_{1} u^{a_{1}} & (0 \leq u \leq 1), \\
c_{0} u^{a_{1}} \leq G(u) \leq c_{1} u^{a_{0}} & (1 \leq u<\infty) .
\end{array}
$$

Proof. We begin with the right-hand side of (3.4). Let $H=C G(C$ the constant in (1.13)). Then $H$ is continuous and increasing, $H(0)=0$ and

$$
H(u v) \leq H(u) H(v) \quad(u, v \geq 0) .
$$


It follows from (3.6) that for any real number $s$

$$
H(\exp (-s)) \leq H(\exp (-s / n))^{n} \quad(n \geq 1) .
$$

Since $H$ is continuous and vanishes at the origin, we must have, for $\sigma>0$ and sufficiently large, $H(\exp (-\sigma))<1$. For $0<u \leq 1$ there exists $n \geq 1$ such that $\exp (-\sigma n) \leq u \leq$ $\exp (\sigma(n-1))$. Then

$$
\begin{aligned}
H(u) & \leq H(\exp (-\sigma(n-1))) \leq H(\exp (-\sigma)) n-1 \leq H(\exp (-\sigma))^{-\log u / \sigma} \\
& =\exp \left(a_{1} \log a\right)=u^{a_{1}},
\end{aligned}
$$

where

$$
a_{1}=\frac{-\log H(\exp (-\sigma))}{\sigma}>0 .
$$

This immediately establishes the right-hand side of (3.4). As for the left-hand side of (3.4), observe that $0<H(1) \leq H\left(e^{n}\right) H\left(e^{-n}\right) \leq H(e)^{n} H\left(e^{-n}\right)$ and that $H\left(e^{-n}\right) \rightarrow 0$ as $n \rightarrow \infty$; thus $H(e)>1$. Let $u, n$ be as before, with $\sigma$ replaced by 1 :

$$
\begin{aligned}
H(u) & \geq H\left(e^{-n}\right) \geq H(1) H(e)^{-n} \geq H(1) H(e)^{(\log u-1)} \\
& =H(1) \exp \left(a_{0}(\log u-1)\right)=\frac{H(1)}{\exp \left(a_{0}\right)} u^{a_{0}},
\end{aligned}
$$

where $a_{0}=\log H(e)>0$. To prove (3.5) we proceed in a rather similar way. Let $u \geq 1$ and choose $n \geq 1$ in such a way that

$$
e^{n-1} \leq u \leq e^{n}
$$

Then

$$
H(u) \leq H\left(e^{n}\right) \leq H(e)^{n} \leq \exp \left(a_{0}(\log u+1)\right)=\exp \left(a_{0}\right) .
$$

On the other hand, with $\exp \sigma(n-1)) \leq u \leq \exp \sigma n)$ we have

$$
H(1) \leq H\left(\operatorname { e x p } ( \sigma ( n - 1 ) ) H \left(\exp (-\sigma(n-1)) \leq H\left(\exp (\sigma(n-1)) H(\exp (-\sigma))^{n+1}\right.\right.\right.
$$

so that

$$
\begin{aligned}
H(u) & \geq H(\exp (\sigma(n-1))) \geq H(1) H(\exp (-\sigma))^{-(n-1)} \\
& =H(1) \exp \left(a_{1}(\log u-1)\right)=\frac{H(1)}{\exp \left(a_{1}\right)} u^{a_{1}} .
\end{aligned}
$$

This ends the proof of (3.5) and of Lemma 3.2.

It is natural to ask whether hypothesis (1.13) is a consequence of the other assumptions in Theorem 1.2. The answer is trivially in the negative; for an immediate counterexample is $F(v)=e^{v}-1(v \geq 0)$; in fact, its inverse function $G(u)=\log (1+u)$ fails to satisfy the left-hand side of (3.5) for any positive constants $d_{0}, c_{0}$. A less trivial task is to construct a function satisfying all the assumptions in Theorem 2 plus inequalities of the type of (3.4), (3.5) but failing to satisfy (1.13). One such example can be constructed as follows. Let

$$
\hat{F}(v)=v^{1+(\log v)-1 / 2} \quad(v>0) .
$$


We have

$$
\hat{F}^{\prime}\left(e^{y}\right)=e^{-y} \frac{d}{d y} \hat{F}\left(e^{y}\right)=e^{-y} \frac{d}{d y}\left(e^{y+y^{2} / 2}\right)=e^{-y}\left(e^{y+y^{1 / 2}}\right)\left(1+\frac{1}{2} y^{-1 / 2}\right)=e^{y^{1 / 2}}\left(1+\frac{1}{2} y^{-1 / 2}\right) .
$$

From this it is clear that there exists a $v_{0}>1$ such that $\hat{F}^{\prime}\left(v_{0}\right)>0$ and $\hat{F}^{\prime}$ is monotone increasing (hence $\hat{F}$ is convex) in $\left[v_{0}, \infty\right)$. Let

$$
\begin{aligned}
\tilde{F}(v) & =\hat{F}(v) \quad\left(v \geq v_{0}\right) \\
& =\hat{F}\left(v_{0}\right)+\hat{F}^{\prime}\left(v_{0}\right)\left(v-v_{0}\right) \quad\left(v \leq v_{0}\right)
\end{aligned}
$$

and

$$
F(v)=\tilde{F}(v)-\widetilde{F}(0) \quad(v \geq 0) .
$$

Then it is clear that $F$ is convex and strictly increasing, $F(0)=0$. As for condition (1.12), we have

$$
\int_{v_{0}}^{\infty} \frac{d v}{F(v)}=\int_{v_{0}}^{\infty} \frac{d v}{v^{1+(\log v)^{-1 / 2}}-\tilde{F}(0)} .
$$

Through the change of variables $v=e^{y}$, this integral becomes

$$
\int_{\log _{v_{0}}}^{\infty} \frac{e^{y} d y}{e^{y+y^{1 / 2}}-\widetilde{F}(0)}=\int_{\log _{0} v_{0}}^{\infty} \frac{d y}{e^{y^{1 / 2}}-\widetilde{F}(0) e^{-y}}<\infty .
$$

Thus, (1.12) is satisfied.

It is not difficult to verify that $G$ satisfies (3.4), (3.5) for $a_{0}>1, a_{1}<1$ and convenient $b_{0}, b_{1}, c_{0}, c_{1}>0$.

Let $v \geq v_{0}$. It follows from the definition (3.7)-(3.8)-(3.9) of $F$ that

$$
F\left(\frac{1}{v}\right)=\hat{F}^{\prime}\left(v_{0}\right) \frac{1}{v}, \quad F(v)=v^{1+(\log v)-1 / 2}-\widetilde{F}(0) .
$$

Thus,

$$
F\left(\frac{1}{v}\right) F(v)=\hat{F}^{\prime}\left(v_{0}\right)\left[v^{(\log v)^{-1 / 2}}-\frac{\widetilde{F}(0)}{v}\right] .
$$

Clearly $\lim _{v \rightarrow \infty} F(1 / v) F(v)=\infty$, whereas $F(1 / v \cdot v)=F(1)$. Accordingly, for arbitrarily large constants $C$

$$
F\left(\frac{1}{v}\right) F(v)>C F(1)
$$

for $v$ large enough.

Let $w=F(1 / v), z=F(v)$. Then

$$
G(w) G(z)=G\left(F\left(\frac{1}{v}\right)\right) G(F(v))=\frac{1}{v} v=1 .
$$

Applying the monotone increasing function $G$ to both sides of (3.10), we have

$$
G(w z)>G(C F(1))=G(C F(1)) G(w) G(z)
$$

because of (3.11). Since $\lim _{v \rightarrow \infty} G(v)=\infty, G(C F(1))$ becomes arbitrarily large as $c \rightarrow \infty$. This shows that condition (1.13) cannot hold for $G$. 
It is a rather interesting question whether an estimate of the form (1.16) would still hold if (1.13) is replaced by a less stringent condition. That some such condition is necessary is shown as follows. Let $F$ be a positive function defined in $v \geq 0$ and let $\left\{g_{n}\right\}$ be any sequence in $L^{2}(0, \infty)$, biorthogonal to $\{\exp [-F(k) t]\}, k \geq 1$. Assume that an inequality of the type of (1.16) takes place. Then,

$$
\left\|q_{n}\right\|_{L^{2}(0, \infty)} \leq \exp (K n) \quad(n \geq 1) .
$$

However,

$$
1=\int_{0}^{\infty} q_{n}(t) e^{-F(n) t} d t \leq[2 F(n)]^{-1 / 2}\left\|q_{n}\right\|_{L^{2}(0, \infty)}
$$

which, together with (3.8), implies

$$
F(n) \leq \frac{1}{2} \exp (2 K n) \quad(n \geq 1) .
$$

Somewhere between (1.13) and (3.13) one might hope to find a condition both necessary and sufficient, together with (1.11) and (1.12), for the validity of Theorem 1.2 but no such condition is known to the authors at this writing.

We continue with some more results needed in the proof of Theorem 2.

The counting function $N(u)$ for a sequence $\Lambda=\left\{\lambda_{k}\right\}$ is defined by

$$
\begin{array}{ll}
N(u)=0 \quad\left(u<\lambda_{1}\right) \\
N(u)=k \quad\left(\lambda_{k} \leq u<\lambda_{k+1}\right), \quad k=1,2,3 \cdots .
\end{array}
$$

Imbedding Lemma 3.3. Let $\Lambda$ be a sequence satisfying the conditions in Theorem 1.2. Then there exists a sequence $M=\left\{\mu_{i}\right\}$ such that

(a) $\mu_{1}=F(l)$

(b) for each $\lambda_{k}$ there exists a $j(k)$ such that

$$
\mu_{j(k)}=\lambda_{k} .
$$

(c) If $\hat{N}(u)$ is the counting function for $M$, then

$$
\left|\hat{N}(u)-\frac{G(u)}{l}\right| \leq 1 \quad(0 \leq u<\infty) .
$$

(d) Let $\nu_{i}=G\left(\mu_{i}\right)(j \geq 1)$. Then any subinterval of $[0, \infty)$ of length $\leq l$ contains at most two of the $\nu_{i}$.

Proof. Let $I_{i}(j \geq 1)$ be the intervals defined by

$$
I_{0}=[0, l] \quad I_{i}=((j-1) l, j l], \quad j=1,2,3, \cdots .
$$

Condition (1.15) shows that each $I_{i}$ contains at most one $w_{k(i)}$. If $I_{i}$ contains such a $w_{k(i)}$ we set $\nu_{i}=w_{k(i)}$; if not $\nu_{i}=j l$. Clearly, if $\tilde{N}(v)$ is the counting function of $\left\{v_{i}\right\}$, then

$$
\left|\tilde{N}(v)-\frac{v}{l}\right| \leq 1 \quad(v \geq 0) .
$$

Let now $\mu_{i}=F\left(\nu_{j}\right)(j \geq 1)$. Then $\hat{N}(u)=\tilde{N}(G(u))$ is the counting function for $M$. If we let $v=G(u),(3.1)$ implies that 


$$
\left|\widehat{N}(u)-\frac{G(u)}{l}\right|=\left|\tilde{N}(G(u))-\frac{G(u)}{l}\right| \leq 1 \quad(u \geq 0) .
$$

Parts (b) and (c) of the lemma have thus been proved. Part (a) is an immediate consequence of the fact that $w_{1} \geq l$. Part (d) follows from the fact that each interval of length $\leq l$ meets at most two of the $I_{i}$, each of which contains exactly one $\nu_{i}$.

Imbedding Lemma 3.4. There exists a positive number $\delta$ such that for any positive integer $n$ the sequence $M$ of Lemma 3.3 can be modified in such a way that if $\mu_{l}=\lambda_{n}$ then $\lambda_{n}-\mu_{l-1} \geq \delta, \mu_{l+1}-\lambda_{n} \geq \delta$ and

$$
\left(\lambda_{n} / \mu_{l-1}\right)-1=1-\left(\lambda_{n} / \mu_{l+1}\right) .
$$

Properties (a) and (b) of Lemma 3.3 are retained and property (c) is replaced by

$$
\left|N_{n}(u)-G(u) / l\right| \leq 3 \quad(u \geq 0),
$$

$N_{n}(u)$ being the counting function for the modified sequence $M_{n}$.

Proof: It was observed in Sec. 1 that if $\Lambda \in \mathfrak{T}(F, l)$, there exists a number $\delta_{0}>0$ (independent of $\Lambda$ ) such that

$$
\lambda_{1}>\delta_{0}, \quad \lambda_{k+1}-\lambda_{k}>\delta_{0} \quad(k=1,2, \cdots) .
$$

Take $\delta \leq \delta_{0} / 2$ and set

$$
\mu=\lambda_{n}+\delta, \quad \tilde{\mu}=\lambda_{n}-\tilde{\delta},
$$

where $\tilde{\delta}$ is determined so that

$$
\frac{\lambda_{n}}{\tilde{\mu}}-1=\frac{\lambda_{n}}{\lambda_{n}-\tilde{\delta}}-1=1-\frac{\lambda_{n}}{\lambda_{n}+\delta}=1-\frac{\lambda_{n}}{\mu} .
$$

This is true if and only if

$$
\frac{\tilde{\delta}}{\lambda_{n}-\tilde{\delta}}=\frac{\delta}{\lambda_{n}+\delta},
$$

from which it is clear that exactly one such $\tilde{\delta}$ exists and $\tilde{\delta}<\delta$.

Let $w=G(\mu), w=G(\tilde{\delta})$. Since $G$ is concave, for every $u_{0}>0$ there exists a positive $\gamma$ such that

$$
|G(u)-G(v)| \leq \gamma|u-v|, \quad\left(u, v \geq u_{0}>0\right) .
$$

Then, as $\tilde{\mu}=\lambda_{n}-\tilde{\delta}=F\left(w_{n}\right)-\tilde{\delta}>F\left(w_{1}\right)-\delta_{0}>0$,

$$
w-\tilde{w} \leq \gamma(\mu-\tilde{\mu})<2 \gamma \delta .
$$

We reduce $\delta$ further, if necessary, so that

$$
0<2 \gamma \delta<l \text {. }
$$

Then, from (d) of Lemma 3.3, the interval $[\tilde{w}, w]$ contains at most one $\nu_{j}$ in addition to $\nu_{l}=w_{n}$. It is clear from (3.20), (3.21) and (1.15) that $\nu_{i}$ is not one of the $w_{k}$. If $w \leq$ $\nu_{i}<w_{n}=\nu_{l}$ then $j=l-1$. We discard $\nu_{j}=\nu_{l-1}$ and rename $\tilde{w}$ as $\nu_{l-1}$. Then we rename $w$ as $\nu_{l+1}$ and $\nu_{k}$ as $\nu_{k+1}$ for $k>l$. This is illustrated as follows $\left(\widetilde{M}_{n}\right.$ denotes the modified sequence): 


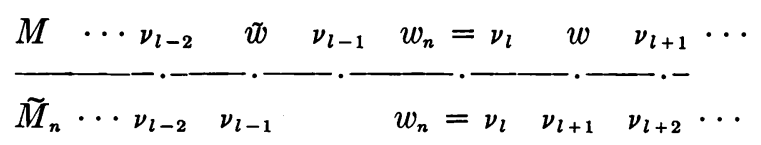

Similar renamings are employed if $w_{n}<\nu_{i} \leq w$ or if $[\tilde{w}, w]$ contains no $\nu_{i}$ other than $\nu_{l}=w_{n}$. These two cases can be visualized as follows.

$$
\begin{aligned}
& M \quad \cdots \nu_{l-1} \quad \tilde{w} \quad w_{n}=\nu_{l} \quad \nu_{l+1} \quad w \quad \nu_{l+2} \cdots \\
& \overline{\tilde{M}_{n} \cdots \nu_{l-1} \quad \nu_{l} \quad \nu_{l+1}} \cdot-\cdot-\cdot- \\
& M \quad \cdots \nu_{l-1} \quad \tilde{w} \quad w_{n}=\nu_{l} \quad w \quad \nu_{l+1} \\
& \overline{\tilde{M}_{n} \cdots \nu_{l-1}} \quad \nu_{l} \quad \nu_{l+1} \quad \nu_{l+2} \quad \nu_{l+3}
\end{aligned}
$$

It is plain that, if $\tilde{N}_{n}(u)$ is the counting function of the modified sequence $\tilde{M}_{n}$, then we have

$$
\left|\hat{N}(u)-\tilde{N}_{n}(u)\right| \leq 1 \quad(u \geq 0)
$$

$\left(\hat{N}\right.$ the counting function of the sequence $\left.\left\{\nu_{i}\right\}\right)$ whenever the interval $[\tilde{w}, w]$ contains a $\nu_{i}$ other than $\nu_{l}$; otherwise,

$$
\left|\hat{N}(u)-\tilde{N}_{n}(u)\right| \leq 2 \quad(u \geq 0) .
$$

It is then clear from (3.16) that (3.19) is satisfied. The fact that (a) and (b) of Lemma 3.3 still hold is evident.

We conclude by defining $\mu_{0}=F(l)-\delta_{0}$. Clearly $0<\mu_{0}<\mu_{k}(k=1,2, \cdots)$. If

$$
M_{n}=\tilde{M}_{n} \cup\left\{\mu_{0}\right\}
$$

and $N_{n}$ is the counting function of $M_{n}$, then $\left|N_{n}(u)-\tilde{N}_{n}(u)\right| \leq 1$; this combined with (3.16) yields

$$
\left|N_{n}(u)-G(u) / l\right| \leq 4
$$

4. Proof of Theorem 1.2 (continuation). We proceed now to estimate $\left\|q_{n}\right\|$ according to the formula (1.5). It is clear that $\left\|p_{n}-r_{n}\right\|$, the distance from $p_{n}$ to $E(n, \Lambda, \infty)$ must be at least as large as the distance $\left\|p_{n}-\hat{r}_{n}\right\|$, where $\hat{r}_{n}$ is the closest point to $p_{n}$ lying in the closed subspace $E\left(l, M_{n}, \infty\right)$ spanned by the functions exp $\left(-\mu_{i} t\right), j \neq l$, where $M_{n}=\left\{\mu_{i}\right\}$ is the sequence constructed in Lemmas 3.3 and 3.4 and the paragraphs thereafter. Then $\left\|q_{n}\right\|_{L^{2}(0, \infty)}$ is majorized by a formula similar to (2.1):

$$
\left\|q_{n}\right\| \leq \frac{1}{\left\|p_{n}-\hat{r}_{n}\right\|}=\left(\frac{\lambda_{n}}{2}\right)^{1 / 2} \frac{\prod_{i=0}^{\infty}\left(1+\frac{\lambda_{n}}{u_{i}}\right)}{\prod_{i=0}^{\infty}\left|1-\frac{\lambda_{n}}{u_{i}}\right|}
$$

where $\Pi^{\prime}$ indicates that the term $j=l\left(\mu_{l}=\lambda_{n}\right)$ should be omitted.

In order to obtain an upper bound for $\left\|q_{n}\right\|$ we must obtain estimates on the infinite products in (4.1). We shall carry out these estimates in much the same way as corresponding estimates were obtained in [6], to which paper the reader is referred for details. 
We begin with the easier infinite product in the numerator. We have

$$
\begin{aligned}
\log \left[\prod_{i=0}^{\infty}(1\right. & \left.\left.+\frac{\lambda_{n}}{\mu_{i}}\right)\right]=\sum_{i=0}^{\infty} \log \left(1+\frac{\lambda_{n}}{\mu_{i}}\right)=\int_{\mu_{0}-}^{\infty} \log \left(1+\frac{\lambda_{n}}{u}\right) d N_{n}(u) \\
= & {\left[\log \left(1+\frac{\lambda_{n}}{u}\right) N_{n}(u)\right]_{\mu_{0}-}^{\infty}+\lambda_{n} \int_{\mu_{0}}^{\infty} \frac{N_{n}(u) d u}{u\left(u+\lambda_{n}\right)}=\lambda_{n} \int_{\mu_{0}}^{\infty} \frac{N_{n}(u) d u}{u\left(u+\lambda_{n}\right)} . }
\end{aligned}
$$

The vanishing of the boundary terms follows, on the one hand, from the fact that $N_{n}\left(\mu_{0-}\right)=0$. On the other hand (according to Lemma 3.1 (b)), $G(u) / u \rightarrow 0$ as $u \rightarrow \infty$, so that $N_{n}(u) / u \rightarrow 0$ as $u \rightarrow \infty$. Since $\log \left(1+\left(\lambda_{n} / u\right)\right)=O(1 / u)$ as $u \rightarrow \infty$, we have

$$
\log \left(1+\left(\lambda_{n} / u\right)\right) N_{n}(u) \rightarrow 0 \text { as } u \rightarrow \infty .
$$

Now, it follows from (3.22) that

$$
\begin{aligned}
\mid \lambda_{n} \int_{\mu_{0}}^{\infty} \frac{N_{n}(u) d u}{u\left(u+\lambda_{n}\right)}-\frac{\lambda_{n}}{l} & \int_{\mu_{0}}^{\infty} \frac{G(u) d u}{u\left(u+\lambda_{n}\right)} \mid \\
& \leq 4 \lambda_{n} \int_{\mu_{0}}^{\infty} \frac{d u}{u\left(u+\lambda_{n}\right)}=4 \log \left(1+\frac{\lambda_{n}}{\mu_{0}}\right) \leq 4 \log \left(\frac{2 \lambda_{n}}{\mu_{0}}\right) .
\end{aligned}
$$

This enables us to restrict attention to the second integral between the absolute value signs in (4.2). If we set $u=\lambda_{n} v$, that integral becomes

$$
\frac{1}{l} \int_{\mu_{0} / \lambda_{n}}^{\infty} \frac{G\left(\lambda_{n} v\right) d v}{v(v+1)}
$$

Making use of hypothesis (1.13), this integral can be estimated as follows:

$$
\frac{1}{l} \int_{\mu_{0} / \lambda_{n}}^{\infty} \frac{G\left(\lambda_{n} v\right) d v}{v(v+1)} \leq \frac{C G\left(\lambda_{n}\right)}{l} \int_{\mu_{0} / \lambda_{n}}^{\infty} \frac{G(v) d v}{v(v+1)} \leq \frac{C G\left(\lambda_{n}\right)}{l} \int_{0}^{\infty} \frac{G(v) d v}{v(v+1)}=C_{1} G\left(\lambda_{n}\right),
$$

where

$$
C_{1}=\frac{C}{l} \int_{0}^{\infty} \frac{G(v) d v}{v(v+1)}<\infty
$$

by virtue of Lemma 3.1 and the right-hand inequality (3.2). Putting (4.2), (4.3) and (4.4) together, we obtain

$$
\log \left[\prod_{i=0}^{\infty}\left(1+\frac{\lambda_{n}}{\lambda_{i}}\right)\right] \leq C_{1} G\left(\lambda_{n}\right)+4 \log \left(\frac{2 \lambda_{n}}{\mu_{0}}\right) .
$$

For the infinite product appearing in the denominator of (4.1) we use similar ideas but the work is slightly more complicated. We define

$$
\begin{aligned}
\bar{N}_{n}(u) & =N_{n}(u), 0 \leq u<\mu_{l}=\lambda_{n}, \\
& =N_{n}(u)-1, \mu_{l} \leq u<\infty .
\end{aligned}
$$

Then we have 


$$
\begin{aligned}
\log \left[\prod_{i=0}^{\infty}\left|1-\frac{\lambda_{n}}{\mu_{i}}\right|\right]= & \int_{\mu_{0}-}^{\mu_{l-1}} \log \left(\frac{\lambda_{n}}{u}-1\right) d \bar{N}_{n}(u)+\int_{\mu_{l+1}-}^{\infty} \log \left(1-\frac{\lambda_{n}}{u}\right) d \bar{N}_{n}(u) \\
= & {\left[\bar{N}_{n}(u) \log \left(\frac{\lambda_{n}}{u}-1\right)\right]_{\mu_{0}-}^{\mu_{l-1}}+\left[\bar{N}_{n}(u) \log \left(1-\frac{\lambda_{n}}{u}\right)\right]_{\mu_{l+1-}-}^{\infty} } \\
& +\lambda_{n} \int_{\mu_{0}}^{\mu_{l-1}} \frac{\bar{N}_{n}(u) d u}{u\left(\lambda_{n}-u\right)}+\lambda_{n} \int_{\mu_{l+1}}^{\infty} \frac{\bar{N}_{n}(u) d u}{u\left(\lambda_{n}-u\right)}
\end{aligned}
$$

Observing that $\bar{N}_{n}\left(\mu_{0-}\right)=0, \bar{N}_{n}(u)=o(u)$ as $u \rightarrow \infty$ and combining the equality $\bar{N}_{n}\left(\mu_{l-1}\right)=\bar{N}_{n}\left(\mu_{l+1-}\right)$ with (3.14), we see that the boundary terms cancel out. Now, making use of (3.22) in the same way as in the treatment of the infinite product in (4.2), we see that

$$
\log \left|\prod_{i=0}^{\infty}\left(1-\frac{\lambda_{n}}{\mu_{j}}\right)\right|=\frac{\lambda_{n}}{l} \int_{\mu_{0}}^{\mu_{l-1}} \frac{G(u) d u}{u\left(\lambda_{n}-u\right)}+\frac{\lambda_{n}}{l} \int_{\mu_{l+1}}^{\infty} \frac{G(u) d u}{u\left(\lambda_{n}-u\right)}+P,
$$

where

$$
\begin{aligned}
|P| \leq 3 \lambda_{n}\left[\int_{\mu_{0}}^{\mu_{l-1}} \frac{d u}{u\left(\lambda_{n}-u\right)}+\int_{\mu_{l+1}}^{\infty} \frac{d u}{u\left(u-\lambda_{n}\right)}\right] \\
\quad=3\left[\log \left(\frac{\lambda_{n}}{\mu_{0}}-1\right)-\log \left(\frac{\lambda_{n}}{\mu_{l-1}}-1\right)-\log \left(1-\frac{\lambda_{n}}{\mu_{l+1}}\right)\right] \\
\leq 3\left[\log \left(\frac{\lambda_{n}}{\mu_{0}}\right)-2 \log \left(1+\frac{\lambda_{n}}{\mu_{l+1}}\right)\right] \\
\quad=3\left[\log \left(\frac{\lambda_{n}}{\mu_{0}}\right)+2 \log \left(\frac{\lambda_{n}+\delta}{\delta}\right)\right] \leq C_{2} \log \lambda_{n} .
\end{aligned}
$$

We observe here that $\mu_{0}$ (see Sec. 3) and $\delta$ are chosen in a way depending only on $F$ and $l$, so that (4.9) holds for any $\Lambda \in \mathfrak{L}(F, l)$.

We turn now to the integrals involving $G$ in the right-hand side of (4.8). Setting $u=\lambda_{n} v$, we obtain

$$
\begin{aligned}
& \frac{\lambda_{n}}{l} \int_{\mu_{0}}^{\mu_{l-1}} \frac{G(u) d u}{u\left(\lambda_{n}-u\right)}+\frac{\lambda_{n}}{l} \int_{\mu_{l+1}}^{\infty} \frac{G(u) d u}{u\left(\lambda_{n}-u\right)} \\
& \quad=\frac{1}{l} \int_{\mu_{0} / \lambda_{n}}^{\mu_{l-1} / \lambda_{n}} \frac{G\left(\lambda_{n} v\right) d v}{v(1-v)}+\frac{1}{l} \int_{\mu_{l+1} / \lambda_{n}}^{\infty} \frac{G\left(\lambda_{n} v\right) d v}{v(1-v)} .
\end{aligned}
$$

To estimate these integrals, we need some more information about $G$. Observe that if $w>0$ then it follows immediately from the fact that $G$ is concave and increasing that

$$
(G(w) / w)(u-w) \leq G(u)-G(w) \leq 0 \quad(0 \leq u \leq w) .
$$

On the other hand, we must have

$$
(G(w) / w)(u-w) \geq G(u)-G(w) \geq 0 \quad(u \geq w) .
$$

In fact, if $G(u)>G(w)+(G(w) / w)(u-w)$ for some $u>w$ then the chord joining the origin and $(u, G(u))$ would have points of the graph of $G$ (namely, $(w, G(w))$ lying below it, which would contradict the concavity of $G$. Putting (4.11) and (4.12) together, we see that 


$$
G(u)-G(w)=(G(w) / w)(u-w) H(u, w) \quad(u, w \geq 0)
$$

where

$$
0 \leq H(u, w) \leq 1 \quad(u, w \geq 0) .
$$

We shall use (4.13) in a slightly different form: setting $u=\lambda_{n} v, w=\lambda_{n}$, we get

$$
G\left(\lambda_{n} v\right)=G\left(\lambda_{n}\right)+G\left(\lambda_{n}\right)(v-1) H\left(\lambda_{n} v, \lambda_{n}\right) .
$$

Let $\rho=\mu_{0} / F(l)=1-\delta_{0} / F(l)$. Then, since $\lambda_{n} \geq F(l)$ for all $n$,

$$
\frac{\mu_{0}}{\lambda_{n}} \leq \rho<1-\frac{\delta_{0}}{2 F(l)} \leq 1-\frac{\delta}{F(l)} \leq 1-\frac{\delta}{\lambda_{n}}=\frac{\mu_{l-1}}{\lambda_{n}} .
$$

Next let $R=1+\delta_{0} / 2 F(l) ;$ then

$$
R>1+\frac{\delta}{F(l)} \geq 1+\frac{\delta}{\lambda_{n}}=\frac{\mu_{l+1}}{\lambda_{n}} .
$$

We can then write

$$
\begin{aligned}
\frac{1}{l} \int_{\mu_{0} / \lambda_{n}}^{\mu l-1 / \lambda_{n}} \frac{G\left(\lambda_{n} v\right) d v}{v(1-v)}+\frac{1}{l} \int_{\mu_{l+1} / \lambda_{n}}^{\infty} \frac{G\left(\lambda_{n} v\right) d v}{v(1-v)} & \\
= & \frac{1}{l} \int_{\mu_{0} / \lambda_{n}}^{\rho} \frac{G\left(\lambda_{n} v\right) d v}{v(1-v)}+\frac{1}{l} \int_{\rho}^{\mu_{l-1 / \lambda_{n}}} \frac{G\left(\lambda_{n} v\right) d v}{v(1-v)} \\
& +\int_{\mu_{l+1} / \lambda_{n}}^{R} \frac{G\left(\lambda_{n} v\right) d v}{v(1-v)}+\int_{R}^{\infty} \frac{G\left(\lambda_{n} v\right) d v}{v(1-v)} .
\end{aligned}
$$

Since we need to estimate (4.16) from below, we may discard the first integral, which is positive. The last, which is negative, can be estimated as follows:

$$
\frac{1}{l} \int_{R}^{\infty} \frac{G\left(\lambda_{n} v\right) d v}{v(1-v)} \geq-C_{3} G\left(\lambda_{n}\right),
$$

where

$$
C_{3}=\frac{C}{l} \int_{R}^{\infty} \frac{G(v) d v}{v(v-1)}<\infty
$$

by virtue of Lemma 3.1. The second and third integrals will be estimated with the aid of (4.15) and (4.14). We have

$$
\begin{aligned}
\frac{1}{l} \int_{\rho}^{\mu l-1 / \lambda_{n}} & \frac{G\left(\lambda_{n} v\right) d v}{v(1-v)}+\frac{1}{l} \int_{\mu_{l+1} / \lambda_{n}}^{R} \frac{G\left(\lambda_{n} v\right) d v}{v(1-v)} \\
= & \frac{G\left(\lambda_{n}\right)}{l}\left[\int_{\rho}^{\mu l-1 / \lambda_{n}} \frac{d v}{v(1-v)}+\int_{\mu l+1 / \lambda_{n}}^{R} \frac{d v}{v(1-v)}\right] \\
& -\frac{G\left(\lambda_{n}\right)}{l}\left[\int_{\rho}^{\mu l-1 / \lambda_{n}} \frac{H\left(\lambda_{n} v, \lambda_{n}\right)}{v} d v+\int_{\mu_{l+1} / \lambda_{n}}^{\infty} \frac{H\left(\lambda_{n} v, \lambda_{n}\right)}{v} d v\right] \\
\geq & \frac{G\left(\lambda_{n}\right)}{l}\left\{\left[\log \left(\frac{v}{1-v}\right)\right]_{\rho}^{\mu l-1 / \lambda_{n}}+\left[\log \left(\frac{v}{v-1}\right)\right]_{\mu l+1 / \lambda_{n}}^{R}\right\}-\frac{G\left(\lambda_{n}\right)}{l} \int_{\rho}^{R} \frac{d v}{v}
\end{aligned}
$$




$$
\begin{aligned}
& =\frac{G\left(\lambda_{n}\right)}{l}\left[\log \frac{1-\rho}{\rho}+\log \frac{R}{R-1}-\log R+\log \rho\right] \\
& =-\frac{1}{l} \log \frac{R-1}{1-\rho} G\left(\lambda_{n}\right)=-C_{4} G\left(\lambda_{n}\right) .
\end{aligned}
$$

Putting together (4.9), (4.17) and (4.18), we obtain

$$
\log \left[\prod_{i=0}^{\infty}\left|1-\frac{\lambda_{n}}{\lambda_{i}}\right|\right] \geq-C_{2} \log \lambda_{n}-\left(C_{3}+C_{4}\right) G\left(\lambda_{n}\right) .
$$

We obtain from the first inequality in (3.5) that

$$
\log \lambda_{n} \leq C_{5} G\left(\lambda_{n}\right) \quad(n=1,2, \cdots)
$$

where $C_{5}>0$. Putting together (4.1), (4.6), (4.19) and (4.20), we plainly obtain an estimate of the form (1.16). This ends the proof of Theorem 1.2.

5. Proof of Theorem 1.3. We shall show that if Theorem 1.3 does not hold we are led to a contradiction. Assume that for some $T>0$ there exists an infinite collection $\left\{\Lambda_{m}\right\}$, $\Lambda_{m}=\left\{\lambda_{m n}\right\}$ of sequences in $\mathfrak{L}(\rho, \Re)$ and corresponding exponential polynomials

$$
P_{m}(t)=\sum_{n=1}^{N(m)} a_{m n} \exp \left(-\lambda_{m n} t\right)
$$

such that

$$
\left\|P_{m}\right\|_{L^{2}(0, T)} \rightarrow 0, \quad m \rightarrow \infty,
$$

while

$$
\left\|P_{m}\right\|_{L^{2}(0, \infty)}=1 \quad(m \geq 1) .
$$

For each $m \geq 1$ let $\left\{q_{m n}\right\}$ be the biorthogonal sequence for the functions $p_{k}(t)=$ exp $\left(-\lambda_{m k} t\right)$ provided by Theorem 1.1. Multiplying $P_{m}(t)$ by $q_{m n}(t)$ and integrating in $(0, \infty)$, we obtain, with the aid of (1.10) and of the Schwarz inequality that, for any $\epsilon>0$,

$$
\left|a_{m n}\right| \leq K(\epsilon) \exp \left(\epsilon \lambda_{m n}\right)\left\|P_{m}\right\|_{L^{2}(0, \infty)}=K(\epsilon) \exp \left(\epsilon \lambda_{m n}\right),
$$

$K(\epsilon)$ the constant in (1.10). But then, if $z$ is any complex number such that $\operatorname{Re} z \geq 2 \epsilon$, we have

$$
\begin{aligned}
\left|P_{m}(z)\right| & \leq \sum_{n=1}^{N(m)}\left|a_{m n}\right| \exp \left(-\operatorname{Re} z \lambda_{m n}\right) \\
& \leq K(\epsilon) \sum_{n=1}^{\infty} \exp \left[-(\operatorname{Re} z-\epsilon) \lambda_{m n}\right] \\
& \leq K(\epsilon) \sum_{n=1}^{\infty} \exp [-\epsilon(n-1) \rho] \exp [-\rho(\operatorname{Re} z-\epsilon)] \\
& =\widetilde{K}(\epsilon) \exp [-\rho(\operatorname{Re} z-\epsilon)]
\end{aligned}
$$

where we have used the fact that $\lambda_{m n} \geq n \rho(m, n=1,2, \cdots)$. Using Montel's theorem on normal families of holomorphic functions together with the estimate (5.3) and the 
Lebesgue dominated-convergence theorem, one readily shows that, if necessary passing to a subsequence,

$$
\lim _{m \rightarrow \infty}\left\|P_{m}-P\right\|_{L^{2}(2 \epsilon, \infty)}=0
$$

where $P(z)$ is a function holomorphic in the halfplane $\operatorname{Re} z \geq 2 \epsilon$. We now make use of (5.4) with $\epsilon<T / 2$. Taking (5.1) into consideration, we see that

$$
P(t) \equiv 0 \quad(2 \epsilon \leq t \leq T)
$$

so that $P(z)$ vanishes identically in $\operatorname{Re} z \geq 2 \epsilon$. Then (5.4) implies

$$
\lim _{m \rightarrow \infty}\left\|P_{m}\right\|_{L^{2}(2 \epsilon, \infty)}=0
$$

which, combined with (5.1) yields

$$
\lim _{m \rightarrow \infty}\left\|P_{m}\right\|_{L^{2}(0, \infty)}=0,
$$

contradicting (5.2). We then conclude that there exists a constant $B=B(\rho, \Re, T)$ such that

$$
\|P\|_{L^{2}(0, \infty)} \leq B\|P\|_{L^{2}(0, T)}
$$

for any exponential polynomial

$$
P(t)=\sum_{n=1}^{N} a_{n} \exp \left(-\lambda_{n} t\right), \quad \Lambda=\left\{\lambda_{n}\right\} \in \mathcal{L}(\rho, \mathfrak{T}) .
$$

Since such polynomials are dense in $E(\Lambda, \infty)$, Theorem 1.3 follows.

6. A control problem. We consider the parabolic partial differential equation

$$
\frac{\partial u}{\partial t}=\kappa \Delta u=\kappa \sum_{i=1}^{p} \frac{\partial^{2} u}{\partial x_{i}^{2}}
$$

where $u(x, t)$ is a function defined in $S_{R} \otimes[0, T]$ :

$$
S_{R}=\left\{x=\left(x_{1}, \cdots, x_{p}\right) \in E^{p}|| x \mid=\left(x_{1}{ }^{2}+\cdots+x_{p}{ }^{2}\right)^{1 / 2} \leq R\right\} .
$$

The numbers $\kappa, R$ and $T$ are assumed positive and $p \geq 1$. For $p=1,2$ or 3 this equation governs the temperature distribution $u$ in a body with the above geometry and uniform coefficient of heat conductivity $\kappa$.

We assume that $u(x, t)$ also satisfies the boundary condition

$$
u(x, t) \equiv f(x, t)\left((x, t) \in B_{R} \otimes[0, T]\right)
$$

where $B_{R}=\left\{x \in E^{p}|| x \mid=R\right\}$ is the boundary of $S_{R}$ in $E^{p}$. We interpret $f(x, t)$ as a control or steering function by means of which we may influence the evolution of solutions $u$ of (6.1). For $p=1,2$ or $3, f(x, t)$ represents the boundary temperature distribution imposed by an external heating-cooling device.

The basic existence and uniqueness theorem which we shall employ is

Theorem 6.1. Let $u_{0}=u_{0}(x)$ be a function defined and continuous in $S_{R}$ and let $f=f(x, t)$ be a function defined and continuous in $B_{R} \otimes[0, T]$. Let the compatibility 
condition

$$
u_{0}(x) \equiv f(x, 0) \quad\left(x \in B_{R}\right)
$$

be satisfied. Then there exists in $S_{R} \otimes[0, T]$ a unique solution $u$ of $(6.1),(6.2)$ with

$$
u(x, 0) \equiv u_{0}(x) \quad\left(x \in S_{R}\right) .
$$

This theorem is a particular case of a result stated in Milgram's appendix to [1]. A proof for $p=1$ that can be readily extended to $p>1$ can be found in [11]. We note that the word "solution" in Theorem 6.1 is understood in the classical sense; i.e., $u$ is continuous in the region $S_{R} \otimes[0, T]$ and has derivatives of all orders in the interior of this region.

In what follows we shall study the following controllability problem. Given, in addition to the initial condition (6.4), a terminal condition

$$
u(x, T)=u_{T}(x) \quad\left(x \in S_{R}\right)
$$

where $u_{T}$ is, say, continuous in $S_{R}$, can we find a function $f$, continuous in $B_{R} \otimes[0, T]$, satisfying (6.3), and such that the solution of (6.1), (6.2), (6.4) satisfies (6.5)?

We derive below conditions on $u_{0}, u_{T}$ sufficient for solution of the above controllability problem. We begin with a very elementary decomposition. Let $f_{0}(x, t)$ be a continuous function defined on $B_{R} \otimes[0, T]$ such that

$$
f_{0}(x, 0) \equiv u_{0}(x) \quad\left(x \in B_{R}\right)
$$

and also

$$
f_{0}(x, t) \equiv 0 \quad\left(x \in B_{R}, t \in[T / 2, T]\right) .
$$

Let $u_{0}(x, t)$ satisfy (6.1), (6.2) (with $f$ replaced by $f_{0}$ ) and (6.4). Then we can solve our controllability problem if we can find a function $u_{1}(x, t)$ defined on $S_{R} \otimes[0, T]$ and a function $f_{1}(x, t)$ defined on $B_{R} \otimes[0, T]$ such that $u_{1}$ satisfies (6.1), $u_{1}$ and $f_{1}$ together satisfy (6.2),

$$
\begin{array}{ll}
u_{1}(x, 0) \equiv 0 & \left(x \in S_{R}\right), \\
f_{1}(x, 0) \equiv 0 & \left(x \in B_{R}\right),
\end{array}
$$

and

$$
u_{1}(x, T) \equiv \hat{u}_{T}(x) \equiv u_{T}(x)-u_{0}(x, T) \quad\left(x \in S_{R}\right) .
$$

It is clear that under these circumstances $u(x, t) \equiv u_{0}(x, t)+u_{1}(x, t)$ and $f(x, t) \equiv$ $f_{0}(x, t)+f_{1}(x, t)$ provide a solution of the original problem, for (6.1), (6.2), (6.3), (6.4) and (6.5) will all be satisfied. Therefore, we will direct our attention below to the construction of $u_{1}$ and $f_{1}$ satisfying the requirements described here. Also, in the work to follow we shall assume $p \geq 2$. The case $p=1$ has already been studied in [6].

Let $A$ be the operator in $L^{2}\left(S_{R}\right)$ defined by $A u=\Delta u$ (cf. (6.1)), with domain $D$ consisting of all functions $u \in L^{2}\left(S_{R}\right)$ such that $\Delta u$ (understood in the sense of distribution) belongs to $L^{2}\left(S_{R}\right)$ and

$$
u(x) \equiv 0 \quad\left(x \in B_{R}\right) .
$$

It is well known (see [3] or [4]) that $A$ is self-adjoint and that it has pure point spectrum: 
the eigenvalues of $A$ are $\left\{-\lambda_{m n} \mid m=0,1,2, \cdots, n=1,2, \cdots\right\}$, where

$$
\lambda_{m n}=\kappa\left[\frac{\nu_{m}{ }^{(n)}}{R}\right]^{2},
$$

$\nu_{m}{ }^{(1)}, \nu_{m}{ }^{(2)}, \cdots$ being the positive zeros of the Bessel function $J_{\alpha(m, \nu)}$ :

$$
\alpha(m, p)=\left[m(m+p-2)+\left(\frac{p}{2}-1\right)^{2}\right]^{1 / 2} .
$$

Each of these eigenvalues has an eigenspace $\mathfrak{H}_{m n}$ of dimension

$$
\beta(m, p)=\left(\begin{array}{c}
m+p-1 \\
p-1
\end{array}\right)-\left(\begin{array}{c}
m+p-3 \\
p-1
\end{array}\right) \quad(m=1,2, \cdots)
$$

and of dimension $1(\equiv \beta(0, p))$ when $m=0$. An orthonormal basis for $\mathfrak{F}_{m n}$ is given by the formula

$$
\begin{aligned}
& \varphi_{m n k}(x)=K_{m n}|x|^{1-p / 2} J_{\alpha(m, p)}\left(\nu_{m}{ }^{(n)} \frac{|x|}{R}\right) Y_{m k}(x) \\
&(m=0,1, \cdots, n=1,2, \cdots, k=1,2, \cdots, \beta(m, p)),
\end{aligned}
$$

where $Y_{m, 1}, \cdots, Y_{m, \beta(m, n)}$ is an orthonormal basis (with respect to the scalar product in $L^{2}\left(B_{R}\right)$ ) of the space $S_{m, p}$ of $p$-dimensional spherical harmonics of degree $m$. (Recall that a $p$-dimensional spherical harmonic of degree $m$ is a function of the form $|x|^{-m} H_{m}(x)$, $H_{m}$ a homogeneous harmonic polynomial in $x_{1}, \cdots, x_{p}$ of degree $m$.) The normalization constant $K_{m n}$ is given by

$$
K_{m n}=2^{1 / 2} R^{(p-3) / 2}\left[J_{\alpha(m, p)+1}\left(\nu_{m}{ }^{(n)}\right)\right]^{-1} \quad(m \geq 0, n \geq 1) .
$$

The functions (6.14) constitute a complete orthonormal set of eigenfunctions for $A$. (For proofs and further details see [2], [5] and [12].)

To avoid repetition, we shall understand throughout what follows that the indices $m, n, k$ always vary in the ranges indicated in (6.14).

Returning to the controllability problem, we develop $\hat{u}_{T}(x)$ (cf. (6.10)) in Fourier series with respect to the eigenfunctions of $A$, i.e.,

$$
\hat{u}_{T}(x)=\sum_{m, n, k} \hat{\mu}_{m n k} \varphi_{m n k}(x) \text {. }
$$

The function

$$
\left.w_{m n k}(x, t) \equiv \exp \left(\lambda_{m n}(t-T)\right) \phi_{m n k}(x)\right)
$$

satisfies

$$
\frac{\partial w_{m n k}}{\partial t}=-\kappa \Delta w_{m n k} \quad\left((x, t) \in S_{R} \otimes[0, T]\right)
$$

and vanishes identically in $B_{R} \otimes[0, T]$. It then follows from the divergence theorem and (6.8) that

$$
\begin{aligned}
0 & =\int_{S_{R} \otimes[0, T]} w_{m n k}\left(\frac{\partial u_{1}}{\partial t}-\kappa \Delta u_{1}\right) d x d t \\
& =\int_{S_{R}} \hat{u}_{T}(x) \phi_{m n k}(x) d x+\kappa \int_{0}^{T} \int_{B_{R}} \exp \left(\lambda_{m n}(t-T)\right) f_{1}(x, t) \frac{\partial}{\partial \nu} \varphi_{m n k}(x) d \sigma d t,
\end{aligned}
$$


where $\partial / \partial \nu$ and $d \sigma$ indicate the outer normal derivative and the area differential in $B_{R}$, respectively. Now

$$
\frac{\partial}{\partial \nu} \varphi_{m n k}(x)=\frac{2^{1 / 2} \nu_{m}{ }^{(n)}}{R^{3 / 2}} \cdot \frac{J_{\alpha(m, p)}^{\prime}\left(\nu_{m}{ }^{(n)}\right)}{J_{\alpha(m, p)+1}\left(\nu_{m}{ }^{(n)}\right)} Y_{m k}(x)=\frac{2^{1 / 2} \nu_{m}{ }^{(n)}}{R^{3 / 2}} Y_{m k}(x)=\left(\frac{2 \lambda_{m k}}{\kappa R}\right)^{1 / 2} Y_{m k}(x),
$$

the second equality being obtained from [12], p. 45. We can then rewrite (6.16), after replacing $t-T$ by $t$ in the last integral and setting $g(x, t)=f_{1}(x, t-T)$, as

$$
\int_{0}^{T} \exp \left(-\lambda_{m n} t\right) g_{m k}(t) d t=-\left(\frac{R}{2 \kappa \lambda_{m n}}\right)^{1 / 2} \hat{\mu}_{m n k}
$$

where

$$
g_{m k}(t)=\int_{B_{R}} g(x, t) Y_{m k}(x) d \sigma .
$$

Taking into account that the $Y_{m k}$ form an orthonormal basis for $L^{2}\left(B_{R}\right)$ (see [2]), we have, at least formally,

$$
g(x, t)=\sum_{m, k} g_{m k}(t) Y_{m k}(x) .
$$

The infinite collection of moment problems (6.17) (one moment problem for each pair $m, k)$ will be solved as follows. For each fixed $m \geq 0$. Let $\left\{q_{m n}\right\}$ be a sequence of elements of $L^{2}(0, T)$ biorthogonal to $p_{m n}(t)=\exp \left(-\lambda_{m n} t\right), n \geq 1$. Then, at least formally, if we set

$$
g_{m k}(t)=\sum_{n=1}^{\infty} c_{m n k} q_{m n}(t)
$$

where

$$
c_{m n k}=-\left(\frac{R}{2 \kappa \lambda_{m n}}\right)^{1 / 2} \hat{\mu}_{m n k},
$$

$g_{m k}$ is a solution of (6.17) for each $m, k$. We need, then, estimates on the $q_{m k}$ to ensure convergence of (6.19) and (6.20). Although these estimates-in $L^{2}(0, T)$-norm-are provided by Corollary 1.6, it is more convenient for the present purpose to have at our disposal uniform pointwise estimates on $q_{m n}(t)$, which we will obtain by a slightly different application of Corollary 1.6.

We proceed first to prove that the $\lambda_{m n}$ satisfy the conditions of Theorem 1.2. We note that for any $\alpha$, the function $z^{1 / 2} J_{\alpha}(z)$ is annihilated by

$$
\frac{d^{2}}{d z^{2}}+\left(1-\frac{\alpha^{2}-1 / 4}{z^{2}}\right) \text {. }
$$

On the other hand, if $\nu_{\alpha}{ }^{(n)}$ is the $n$th positive zero of $J_{\alpha}$ the function $\sin \left(z-\nu_{\alpha}{ }^{(n)}\right)$ vanishes at $\nu_{\alpha}{ }^{(n)}$ and is annihilated by $\left(d^{2} / d z^{2}\right)+1$. If $\alpha \geq \frac{1}{2}$,

$$
1-\frac{\alpha^{2}-1 / 4}{z^{2}} \leq 1
$$

thus it follows from the Sturm oscillation theorem [4, p. 1462] that $\sin \left(z-v_{\alpha}{ }^{(n)}\right)$ must have a zero in $\left(\nu_{\alpha}{ }^{(n)}, \nu_{\alpha}{ }^{(n+1)}\right)$. This plainly implies that

$$
\nu_{\alpha}{ }^{(n+1)}-\nu_{\alpha}{ }^{(n)}>\pi \quad\left(n \geq 1, \alpha \geq \frac{1}{2}\right) .
$$


The range $\alpha \geq \frac{1}{2}$ takes care of all values of $\alpha(m, p)$ in (6.12) except for the case $p=2$, where $\alpha(0,2)=0$. To settle this case, we observe that

$$
1+\frac{1}{4 z^{2}} \leq 1+\frac{1}{4 \nu_{0}^{(n)}} \quad\left(z \geq \nu_{0}^{(n)}\right) .
$$

Then the function $\sin \left(1+\left(1 / 4 \nu_{0}{ }^{(n)}\right)^{1 / 2}\left(z-\nu_{0}{ }^{(n)}\right)\right.$, which is annihilated by

$$
\left(d^{2} / d z^{2}\right)+1+1 / 4 \nu_{0}^{(n)},
$$

must have a zero in $\left(\nu_{0}^{(n)}, \nu_{0}{ }^{(n+1)}\right)$, which implies that

$$
\nu_{0}^{(n+1)}-\nu_{0}^{(n)} \geq\left(1+\frac{1}{4 \nu_{n}^{(n)}}\right)^{-1 / 2} \pi \geq\left(1+\frac{1}{4 \nu_{0}^{(n)}}\right)^{-1 / 2} \pi .
$$

We observe finally that, for $\alpha \geq 0$

$$
1-\frac{\alpha^{2}-1 / 4}{z^{2}} \leq 1+\frac{1}{4 z^{2}}
$$

so that $z^{1 / 2} J_{0}(z)$ must have a zero in $\left(0, \nu_{\alpha}{ }^{(1)}\right)$; then

$$
\nu_{\alpha}{ }^{(1)} \geq \nu_{0}{ }^{(1)} \quad(\alpha \geq 0) .
$$

(We observe that this last application of the oscillation theorem is slightly nonstandard' as the coefficients in (6.25) become infinite at one of the extremes of the interval; however, a look at the proof in [4] shows that the result still holds in this case.) It is true that much finer information about the zeros of $J_{\alpha}$ can be obtained both by Sturm's theorem and by other methods; the reader is referred to [12] for these results. The crude estimates just obtained are, however, sufficient for our purpose.

Let us define the sequences $\left\{\lambda_{m n}\right\}$ by

$$
\lambda_{m 0}=0, \quad \lambda_{m n}=\kappa\left[\frac{\nu_{m}^{(n)}}{R}\right]^{2} \quad(n \geq 1)
$$

for $m=0,1,2, \cdots$. Let $l_{0}$ be any positive number. Then it is clear from (6.23), (6.24), (6.26) that the sequences $\left\{\lambda_{m n}+l_{0}\right\}$ satisfy the hypotheses of Theorem 1.2. Thus Corollary 1.6 applies to show that for each $m \geq 0$ there exists a sequence $\left\{\tilde{q}_{m n}(t)\right\}$, biorthogonal to $\exp \left(-\lambda_{m n} t\right), n \geq 0$, and such that

$$
\left\|\tilde{q}_{m n}\right\|_{L^{2}(0, T)} \leq B \exp K\left(\lambda_{m n}+l_{0}\right)^{1 / 2} \quad(n \geq 0, m \geq 0),
$$

where the constants $B$ and $K$ are independent of $m$ and $n$. Now define

$$
q_{m n}(t)=\lambda_{m n} \int_{0}^{t} \tilde{q}_{m n}(s) d s \quad(0 \leq t \leq T, m \geq 0, n \geq 1) .
$$

Since $q_{m n}, n \geq 1$ is orthogonal to $\exp \left(-\lambda_{m 0} t\right) \equiv 1$, we have

$$
q_{m n}(0)=q_{m n}(T)=0 \quad(m \geq 0, n \geq 1) .
$$

Thus, integrating by parts,

$$
\int_{0}^{T} q_{m n}(t) \exp \left(-\lambda_{m i} t\right) d t=\int_{0}^{T} \tilde{q}_{m n}(t) \exp \left(-\lambda_{m i} t\right)=\delta_{n i} \quad(n, j \geq 1) .
$$


Accordingly, $\left\{q_{m n}\right\}$ is biorthogonal to $\exp \left(-\lambda_{m n} t\right)$ for $n \geq 1$ and, it follows from (6.27), (6.28) and the fact that $(a+b)^{1 / 2} \leq a^{1 / 2}+b^{1 / 2}$ for $a, b \geq 0$ that

$$
\left|q_{m n}(t)\right| \leq B \exp K \lambda_{m n}{ }^{1 / 2} \quad(0 \leq t \leq T, m \geq 0, n \geq 1),
$$

(where $B$ and $K$ are not necessarily the same as in (6.27)).

Assume now that the coefficients $c_{m n k}$ given by (6.21) satisfy

$$
\left|c_{m n k}\right| \leq M \exp \left(-\widehat{K} \lambda_{m n}{ }^{1 / 2}\right) \quad(m \geq 0, n \geq 1)
$$

for some $M>0$ and $\hat{K}>K$ ( $K$ the constant in (6.30). Then, by virtue of the estimate (6.30), the series (6.20) converges absolutely and uniformly in $[0, T]$ for each $m, k$ and thus the function $g_{m k}$ is a continuous function solving the moment problem (6.17). We have, moreover,

$$
\left|g_{m k}(t)\right| \leq M \sum_{n=1}^{\infty} \exp \left(-(\widehat{K}-K) \lambda_{m n}^{1 / 2}\right)
$$

To ascertain convergence of (6.19) we need pointwise bounds on the normalized spherical harmonics $Y_{m k}$. Such bounds are available and are expressed by the inequality

$$
\left|Y_{m k}(x)\right| \leq C m^{(p-2) / 2} \quad\left(x \in B_{R}\right),
$$

where $C$ may depend on $p$ but not on $m$ or $k$. To see that this is true, consider the function

$$
R(x, y)=\sum_{k=1}^{\beta(m, p)} Y_{m k}(x) Y_{m k}(y) \text {. }
$$

Evidently, $R$ is a reproducing kernel for the space $S_{m, p}$ of $p$-dimensional spherical harmonics of degree $m$; that is, if $Q \in \delta_{m, p}$,

$$
Q(x)=\int_{B_{R}} Q(x) R(x, y) d \sigma_{y},
$$

$d \sigma$ being the surface differential in $B_{R}$. Now, if $w$ is a rotation in $R^{n}$ (an orthogonal transformation with determinant +1$)$, it is easy to see with the aid of the change of variable $y^{\prime}=w y$ that $Q(w x, w y)$ is also a reproducing kernel for $\S_{m, p}$; that is, $Q(w x, w y)$ also satisfies (6.34) for any $Q \in S_{m, p}$. Observe next that rotation of the variable transforms an element of $S_{m, p}$ into another element of $S_{m, p}$; thus, for each fixed $x, S(y)=$ $R(x, y)-R(w x, w y)$ belongs to $S_{m, p}$ and its scalar product with an arbitrary element of $S_{m, p}$ vanishes; then $S(y) \equiv 0$, i.e. $R(x, y) \equiv R(w x, w y)$ for any $x, y \in B_{R}$ and any rotation $w$. Since any two points in $B_{R}$ can be mapped into each other by a rotation, $R(x, x)$ must be a constant:

$$
R(x, x)=\sum_{k=1}^{\beta(m, p)} Y_{m k}^{2}(x)=C_{m} \quad\left(x \in B_{R}, m \geq 0\right) .
$$

Since

$$
\int_{B_{R}} Y_{m k}^{2}(x) d \sigma=1 \quad(m \geq 0, k=1, \cdots, \beta(m, p))
$$

we have

$$
C_{m}=\frac{1}{\sigma} \int_{B_{R}} \sum_{k=1}^{\beta(m, p)} Y_{m k}{ }^{2}(x) d \sigma=\frac{\beta(m, p)}{\sigma}
$$


$\left(\sigma\right.$ the area of $\left.B_{R}\right)$. Thus

$$
\left|Y_{m k}(x)\right| \leq C_{m}^{1 / 2} \leq\left(\frac{\beta(m, p)}{\sigma}\right)^{1 / 2}
$$

from which (6.33) follows from the immediate inequality

$$
\beta(m, p) \leq C m^{p-2}
$$

the constant $C$ being independent of $m$.

It is well known (see [12]) that $\nu_{m}{ }^{(1)}$ is bounded below by a positive multiple of a positive power of $m$. Combined with (6.32), (6.33), (6.35) this implies that the numerical series

$$
\sum_{m=0}^{\infty} \sum_{n=1}^{\infty} m^{3(p-2) / 2} \exp \left[-(\hat{K}-K) \lambda_{m n}{ }^{1 / 2}\right]
$$

is convergent. It follows then that the series (6.19), (6.20) is uniformly convergent for $x \in B_{R}, t \in[0, T]$, since it is then dominated term by term by a constant multiple of (6.36). The sum $g(x, t)$ of (6.19) is therefore a continuous function which, using (6.29), satisfies

$$
g(x, 0) \equiv g(x, T) \equiv 0 \quad\left(x \in B_{R}\right) .
$$

We have shown then, that if $\hat{u}_{T}(x)$ (cf. (6.10)) is given by the expansion (6.15) and if the coefficients $\hat{u}_{m n k}$ are such that (cf. (6.21)), (6.31)

$$
\left(\frac{R}{2 \kappa \lambda_{m n}}\right)^{1 / 2}\left|\hat{\mu}_{m n k}\right| \leq M \exp \left(-K \lambda_{m n}{ }^{1 / 2}\right) \quad(m \geq 0, n \geq 1, k=1,2, \cdots, \beta(m, p)),
$$

then there exists a function $g(x, t)$ given by (6.19), (6.20) such that $f_{1}(x, t)=g(x, T-t)$ satisfies (6.16) for these values of $m, n$ and $k$. It is clear from (6.37) that $f_{1}$ satisfies (6.9).

Since $f_{0}(x, t)$ is continuous and satisfies the consistency condition (6.6), $u_{0}(x, T / 2)$ is continuous for $x \in S_{R}$. This combined with (6.7) is enough to show that if

$$
u_{0}(x, T)=\sum_{m, n, k} \tilde{\mu}_{m n k} \varphi_{m n k}(x)
$$

then there is a constant $M_{0}$ such that

$$
\left|\tilde{\mu}_{m n k}\right| \leq M_{0} \exp \left(-T / 2 \lambda_{m n}\right) \quad(m \geq 0, n \geq 1, k=1,2, \cdots, \beta(m, p)) .
$$

Thus we see that if

$$
u_{T}(x) \equiv \hat{u}_{T}(x)+u_{0}(x, T) \equiv \sum_{m, n, k} \mu_{m n k} \varphi_{m n k}(x)
$$

is such that

$\left(\frac{R}{2 \kappa \lambda_{m n}}\right)^{1 / 2}\left|\mu_{m n k}\right| \leq M_{1} \exp \left(-\hat{K} \lambda_{m n}{ }^{1 / 2}\right) \quad(m \geq 0, n \geq 1, k=1,2, \cdots, \beta(m, p))$

for some $M_{1}>0$, then (6.38) is satisfied by the coefficients $\hat{\mu}_{m n k}=\mu_{m n k}-\tilde{\mu}_{m n k}$ for some constant $M>0$. Therefore we have proved the following controllability result, wherein we take $f(x, t) \equiv f_{0}(x, t)+f_{1}(x, t) x \in B_{R}, t \in[0, T]$.

Theorem 6.2. Let the initial state $u_{0}$ be continuous in $S_{R}$ and let the desired terminal state $u_{T}(x)$ have an expansion (6.39) such that (6.40) is satisfied for some 
$M_{1}>0, \widehat{K}$ being the constant in (6.31). Then there exists a continuous control function $f(x, t)$ which satisfies $(6.3)$ such that the solution $u(x, t)$ of the initial-boundary value problem (6.1), (6.2), (6.4) also satisfies the terminal condition (6.5).

The sufficient condition for controllability (6.40) agrees with the condition obtained in [6] for the case $p=1$. It can easily be shown that this is not a necessary condition. The mathematically attractive goal of a necessary and sufficient condition on $u_{T}(x)$ in order that the controllability problem should have a solution $f(x, t)$ lying in an appropriate space is not yet in sight. Such a result would appear to require a much deeper analysis than the one presented here.

\section{REFERENCES}

[1] L. Bers, F. John and M. Schechter, Partial differential equations, Interscience, New York, 1964

[2] A. P. Calderón, Integrales singulares y sus aplicaciones a ecuaciones diferenciales hiperbólicas, Cursos $y$ Seminarios de Matemática, Fasc. 3, Universidad de Buenos Aires, 1960

[3] R. Courant and D. Hilbert, Methods of mathematical physics, Interscience, New York, 1953

[4] N. Dunford and J. T. Schwartz, Linear operators, Part II: Spectral theory, Interscience, New York, 1963

[5] A. Erdélyi (editor), Bateman manuscript project, Vol. II, McGraw-Hill, New York, 1953

[6] H. O. Fattorini and D. L. Russell, Exact controllability theorems for linear parabolic equations in one space dimension, Arch. Rat. Mech. Anal. 4, 272-292 (1971)

[7] S. Kaczmarz and H. Steinhaus, Theorie der Orthogonalreihen, Monografje Matematyczne, Tom VI, Warszawa, 1935

[8] W. A. J. Luxemburg and J. Korevaar, Entire functions and Müntz-Szasz type approximation, Trans. Amer. Math. Soc. 156 (1971)

[9] V. J. Mizel and T. I. Seidman, Observation and prediction for the heat equation, II, Report 70-35, Department of Mathematics, Carnegie-Mellon University

[10] L. Schwartz, Etude des sommes d'exponentielles, 2d edition, Hermann, Paris, 1959

[11] W. Sternberg, Über die Gleichung der Warmeleitung, Math. Ann. 101, 394-398 (1929)

[12] G. N. Watson, A treatise on the theory of Bessel functions, 2d edition, Cambridge University Press, Cambridge, 1944

[13] A. Zygmund, Trigonometric series, 2d edition, Cambridge University Press, Cambridge, 1959 